\title{
APPLICATION OF GAME THEORY IN PLANNING PASSENGER RAIL AND ROAD TRANSPORT ON PARALLEL ROUTES
}

\author{
Svetla Stoilova \\ Technical University of Sofia, Bulgaria \\ stoilova@tu-sofia.bg
}

\begin{abstract}
The game theory provides powerful tools for analysing transport systems and making decisions in situations, where there are opposite interests between the participants. This study proposes a methodology based on the game theory for selection the strategy of passenger transport planning on parallel routes for railway and road transport. The game theory presents the behaviour of the railway and road operators in conflict situations. The methodology uses dual linear programming to assess the strategies. The game is formulated between two transport operators (road and railway) that are competitors in passenger transportation. Two game models have been developed, one based on the profit of the road operator and the other based on the number of passengers. The road operator determines the profit, which is proportional to the flow of passenger traffic through the route. Its purpose is to maximize the overall transport profit. The aim of the railway operator is to minimize the overall transport costs. The research includes four steps. In the first step, the strategies of transportation for railway and road operators are determined. The second step includes formation of the decision matrix and mathematical representation of the problem for both transport operators. The third step gives the optimal strategies for both transport operators. The fourth step verifies the results by using the Hurwitz's coefficient for decision making. The methodology has been approbated for the route Sofia-Burgas from the railway and road network of Bulgaria, which is a part of the Trans-European Transport Network. The strategies for both transport operators have been determined. The game-theoretic formalization presented in the study allows modelling strategies and decision making for both railway and road operators in the case of different interests.
\end{abstract}

Keywords: game theory, decision-making, rail, road, passenger transport, Hurwitz's criterion, transport planning.

\section{Introduction}

The interurban services are usually carried out by rail and road transport. In many cases the routes are parallel. Thus, transport operators in different modes of transport are in competition for attracting passengers. The attracting passengers from one mode of transport are a waste for the other. It is therefore necessary to evaluate different transport alternatives for transport operators, which include transportation planning.

The game theory provides powerful tools for analysing transport systems. It can be used to make decision in situations, where there are opposite interests between the participants. The study proposes a methodology based on the game theory for selection the strategy of passenger transport planning on parallel routes for railway and road transport. The game theory presents the behaviour of the railway and road operators in conflict situations. The methodology uses dual linear programming to assess the strategies. The game theory is a method, which deals with problems with conflicting objectives between two opponents.

The problem of route selection and alternative of transportation has been investigated by various authors.

In [1] a route choice model is elaborated based on the game theory. The proposed model uses alternative routes as game players. The precision of predicted information and familiarity of traffic conditions are applied to build a game. The game theory is applied to assess the transportation systems in cities. The Nash equilibrium is achieved with both pure and mixed strategies for car-pooling system [2]. A problem on public transport management is decided in [3] based on the game theory. The authors treat the passenger flow, transport operator and municipal authorities, which make decisions. The passenger choice is defined between public and private transport by evaluating the value of time. The Nash equilibrium is used to solve the problem. In [4] the game theory is applied to determine the equilibrium between the interests of the population and the capacity of transport infrastructure for urban transport systems.

A game theory approach is applied in [5] to study three public transportation modes conventional bus, taxi and subway (or light rail). A bi-level programming operational model is proposed for decision making. An analysis of the game theory application is done in [6]. The authors 
presented the relations between transport models and games. Four groups of games have been formed: games against a demon (a zero-sum game); games between travellers; games between authorities; and games between travellers and authorities. The game theory and the Nash equilibrium are used in [7] to describe the strategies in urban transport.

In [8] the authors present an application of the game theory. An experiment of the choice of optimal solution on the route of a new bus line is shown in this study. The input into the mathematical model is the data about the connection of the residents of the new settlement to certain stops in the city. In [9] an evolution game model of travel mode choice is proposed. The travels by car, by taxi, by bus, and by rail were considered. The Nash equilibrium is used. The study proposes the different payoff function as a time variable, a monetary variable, both of these, or some other which have been determined by the purposes of the model application. A generalized Nash equilibrium game model is proposed in [10] for describing the passenger route choices and trip modes. A game theoretic approach has been elaborated in [11] to study high speed rail and bus transportation. Two stages of the game have been formulated; one for maximizing the profits of the bus, and other for high speed rail. A twoplayer non-cooperative game is studied in [12] between the network user seeking a path to minimise the expected trip cost and the operator aimed to maximise the expected trip cost. This is described by a two-player, non-cooperative, and zero-sum game with mixed strategy. The Nash equilibrium for this game is used. In [13] the competition of high-speed and conventional rail system in Taiwan, which operated within the parallel network, is studied. A game theoretical model has been developed to describe the rail system pricing strategy.

It can be summed that the game theory is an appropriate method to make decisions in conflict situations. Different game models have been elaborated to study the following problems: alternative routes, traffic planning in the cities public and private transport, traffic planning in the cities high speed rail and bus transportation, high-speed and conventional rail systems.

The purpose of the study is to develop a model for selecting a transport plan for competitive modes of transport, when the service is on parallel routes. A suitable method for this is the game theory.

\section{Materials and methods}

The methodology consists of the following steps.

- Step 1: Determining the strategies of transportation for railway and road operators. The strategies are based on formation of different variants of transport plan for given route.

- Step 2: Formation of a decision (payoff) matrix. The elements of this matrix present the profit (loss) of one transport operator depending on the strategy of the other transport operator.

- Step 3. Compiling a mathematical model based on the game theory and dual linear optimization to make a decision for both transport operators. Determination of the optimal strategies for both transport operators.

- Step 4. Verification of the results by using the Hurwitz's coefficient for decision making.

The game theory is a method, which deals with problems with conflicting objectives between two opponents. In a game conflict, two opponents are named players. Each of them has a number of alternatives or strategies. The game is usually presented by the payoff matrix $\left(a_{i j}\right)_{m \times n}$ :

$$
\left(\begin{array}{cccc}
a_{11} & a_{12} & \ldots & a_{1 n} \\
a_{21} & a_{22} & \ldots & a_{2 n} \\
\ldots & \ldots & \ldots & \ldots \\
a_{m 1} & a_{m 2} & \ldots & a_{m n}
\end{array}\right),
$$

The rows present the strategies for player $A(i=1, \ldots, m)$, the columns present the strategies for plyer $B(j=1, \ldots, n)$. For example, $a_{21}$ expresses the profit of player $\mathrm{A}$, if he chooses his second strategy and player B chooses his first strategy.Such games are known as two-person zero-sum games, because a gain by one player signifies an equal loss to the other. When the two players have several optimal strategies, the game is a mixed strategy game. This game can be presented by the dual linear 
programming method. This means that the optimal solution of one problem (player A) automatically gives the optimal solution of the other (player B).The two problems optimize the same value of the game.

The optimal mixed strategies for player A are:

$$
\begin{gathered}
\max _{x_{i}}\left\{\min \left(\sum_{i=1}^{m} a_{i 1} x_{i}, \sum_{i=1}^{m} a_{i 2} x_{i}, \ldots, \sum_{i=1}^{m} a_{i n} x_{i}\right)\right\}, \\
x_{1}+x_{2}+\ldots+x_{m}=1, \\
0 \leq x_{i} \leq 1, i=1,2, \ldots, m
\end{gathered}
$$

where $x_{i}$-respective probabilities for strategy $A_{i}$ for player A;

Now, let:

$$
\vartheta=\min \left(\sum_{i=1}^{m} a_{i 1} x_{i}, \sum_{i=1}^{m} a_{i 2} x_{i}, \ldots, \sum_{i=1}^{m} a_{i n} x_{i}\right),
$$

The equation implies that

$$
\sum_{i=1}^{m} a_{i j} x_{i} \geq \vartheta, j=1,2, \ldots, n
$$

where $\vartheta$-value of the game.

The problem of player A can be written:

$$
\begin{gathered}
\text { Maximize } \zeta=\vartheta, \\
a_{11} x_{1}+a_{12} x_{2}+\ldots+a_{m 1} x_{m} \leq \vartheta \\
a_{12} x_{1}+a_{22} x_{2}+\ldots+a_{m 2} x_{m} \leq \vartheta \\
\ldots \\
a_{1 n} x_{1}+a_{2 n} x_{2}+\ldots+a_{m n} x_{m} \leq \vartheta \\
x_{1}+x_{2}+\ldots+x_{m}=1,
\end{gathered}
$$

For player B the problem is as follows:

$$
\begin{gathered}
\min _{y_{i}}\left\{\max \left(\sum_{j=1}^{n} a_{j 1} y_{j}, \sum_{j=1}^{n} a_{j 2} y_{j}, \ldots, \sum_{j=1}^{n} a_{m j} y_{j}\right)\right\}, \\
y_{1}+y_{2}+\ldots+y_{n}=1, \\
0 \leq y_{j} \leq 1, j=1,2, \ldots, n .
\end{gathered}
$$

Now, let:

$$
\omega=\max \left(\sum_{j=1}^{n} a_{j 1} y_{j}, \sum_{j=1}^{n} a_{j 2} y_{j}, \ldots, \sum_{j=1}^{n} a_{m j} y_{j}\right),
$$

The problem of player B can be written:

$$
\begin{aligned}
& \text { Minimize } \omega=\vartheta \text {, } \\
& \mid \begin{array}{c}
a_{11} y_{1}+a_{12} y_{2}+\ldots+a_{1 n} y_{n} \leq \vartheta \\
a_{21} y_{1}+a_{22} y_{2}+\ldots+a_{2 n} y_{n} \leq \vartheta \\
\ldots \\
a_{m 1} y_{1}+a_{m 2} y_{2}+\ldots+a_{m n} y_{n} \leq \vartheta
\end{array}, \\
& y_{1}+y_{2}+\ldots+y_{n}=1, \\
& 0 \leq y_{j} \leq 1, j=1,2, \ldots, n \text {. }
\end{aligned}
$$


Transformations for payer $\mathrm{A}$

\begin{tabular}{|c|c|}
\hline Transformation for player A & \multicolumn{1}{c|}{ Game model for player A } \\
\hline Maximize $\zeta=\vartheta=\min (1 / \vartheta)$ & Minimize $\zeta=X_{1}+X_{2}+\ldots+X_{m}$ \\
Subject to & Subject to \\
$a_{11} \frac{x_{1}}{\vartheta}+a_{21} \frac{x_{2}}{\vartheta}+\ldots+a_{m 1} \frac{x_{m}}{\vartheta} \geq 1$ & $a_{11} X_{1}+a_{21} X_{2}+\ldots+a_{m 1} X_{m} \geq 1$ \\
$a_{12} \frac{x_{1}}{\vartheta}+a_{22} \frac{x_{2}}{\vartheta}+\ldots+a_{m 2} \frac{x_{m}}{\vartheta} \geq 1$ & $a_{12} X_{1}+a_{22} X_{2}+\ldots+a_{m 2} X_{m} \geq 1$ \\
$\ldots$ & $\ldots$ \\
$a_{1 n} \frac{x_{1}}{\vartheta}+a_{2 n} \frac{x_{2}}{\vartheta}+\ldots+a_{m n} \frac{x_{m}}{\vartheta} \geq 1$ & $a_{1 n} X_{1}+a_{2 n} X_{2}+\ldots+a_{m n} X_{m} \geq 1$ \\
$\frac{x_{1}}{\vartheta}+\frac{x_{2}}{\vartheta}+\ldots+\frac{x_{m}}{\vartheta}=\frac{1}{\vartheta}$ & $X_{i} \geq 0 ; i=1,2, \ldots, n$ \\
\hline
\end{tabular}

Transformations for payer B

\begin{tabular}{|c|c|}
\hline Transformation for player B & Game model for player B \\
\hline Minimize $\omega=\vartheta=\min (1 / \vartheta)$ & Maximize $\omega=Y_{1}+Y_{2}+\ldots+Y_{n}$ \\
Subject to & Subject to \\
$a_{11} \frac{y_{1}}{\vartheta}+a_{21} \frac{y_{2}}{\vartheta}+\ldots+a_{m 1} \frac{y_{n}}{\vartheta} \geq 1$ & $a_{11} Y_{1}+a_{21} Y_{2}+\ldots+a_{n 1} Y_{n} \leq 1$ \\
$a_{12} \frac{y_{1}}{\vartheta}+a_{22} \frac{y_{2}}{\vartheta}+\ldots+a_{m 2} \frac{y_{n}}{\vartheta} \geq 1$ & $a_{12} Y_{1}+a_{22} Y_{2}+\ldots+a_{n 2} Y_{n} \leq 1$ \\
$\ldots$ & $\ldots$ \\
$a_{1 m} \frac{y_{1}}{\vartheta}+a_{2 m} \frac{y_{2}}{\vartheta}+\ldots+a_{m n} \frac{y_{n}}{\vartheta} \geq 1$ & $a_{1 m} Y_{1}+a_{2 m} Y_{2}+\ldots+a_{m n} Y_{n} \leq 1$ \\
$\frac{y_{1}}{\vartheta}+\frac{y_{2}}{\vartheta}+\ldots+\frac{y_{n}}{\vartheta}=\frac{1}{\vartheta}$ & $Y_{j} \geq 0 ; j=1,2, \ldots, n$ \\
$0 \leq y_{j} \leq 1, j=1,2, \ldots, n$ & $Y_{j}=\frac{y_{j}}{\vartheta} ; \vartheta=\frac{1}{\omega}$ \\
\hline
\end{tabular}

The two problems are transformed to be solved. Table 1and Table 2 present the transformations. The first column of the tables presents the way of transformation; the second column presents the transformed model. The solution of the transformed model gives the results for the game model. The transformed model is solved by dual linear programming.

The Hurwitz's criterion is applied in step 4 to verify the results of the game theory. This criterion uses the coefficient of optimism $\alpha$ that allows making decisions according to different decision approaches. The value of $\alpha$ can be set between 0 and 1 . Generally, $\alpha=0.5$, while $\alpha=1$ represents an optimistic approach, $\alpha=0$ represents a pessimistic approach.

The optimal alternative is determined as, [14]:

$$
\begin{gathered}
H_{i}=\min _{i}\left\{\alpha \min _{j} a_{i j}+(1-\alpha) \max _{j} a_{i j}\right\}, \text { when } a_{i j} \text { presents the costs } \\
H_{i}=\max _{i}\left\{\alpha \max _{j} a_{i j}+(1-\alpha) \min _{j} a_{i j}\right\}, \text { when } a_{i j} \text { presents the profits }
\end{gathered}
$$

\section{Results and discussion}

\section{Determination the strategies for road and railway operators}

The methodology is approbated for the route Sofia-Burgas from the railway and road network of Bulgaria, which is a part of the Trans-European Transport Core Network.

The carriage with road transport by the studied route is realized by three road operators. One road operator mainly operates throughout the day, while the other two have complementary single routes at 
some hours of the day. The player for road transport is presented by all road operators. It is assumed that they are not competitors with each other.

The transportation of passengers for the route Sofia-Burgas is realized by two categories of buses - express buses. The express buses have reduced travel time and do not stop at additional centres along the route, or have only one additional stop.

This study examines the following strategies for road operators: A1- Transportation by buses; A2 - Transportation by express buses; A3 - Transportation by buses and express buses.

In Bulgaria there exist two categories of intercity trains - faster and accelerated fast trains (express). In this study transportation is investigated with a new category of intercity trains - the socalled direct trains. Accelerated fast trains require a reservation and serve large intermediate stations between cities, big transport and important administrative centres. Direct trains also require reservation, but operate between big transport and important administrative centres. The fast trains serve additionally the municipal centres.

This study examines the following strategies for railway operators: R1 - Transportation by accelerate fast trains and fast trains; R2 - Transportation by direct trains, accelerated fast trains and fast trains; R3 - Transportation by direct trains and fast trains. Figure 1 presents the strategies for both operators.

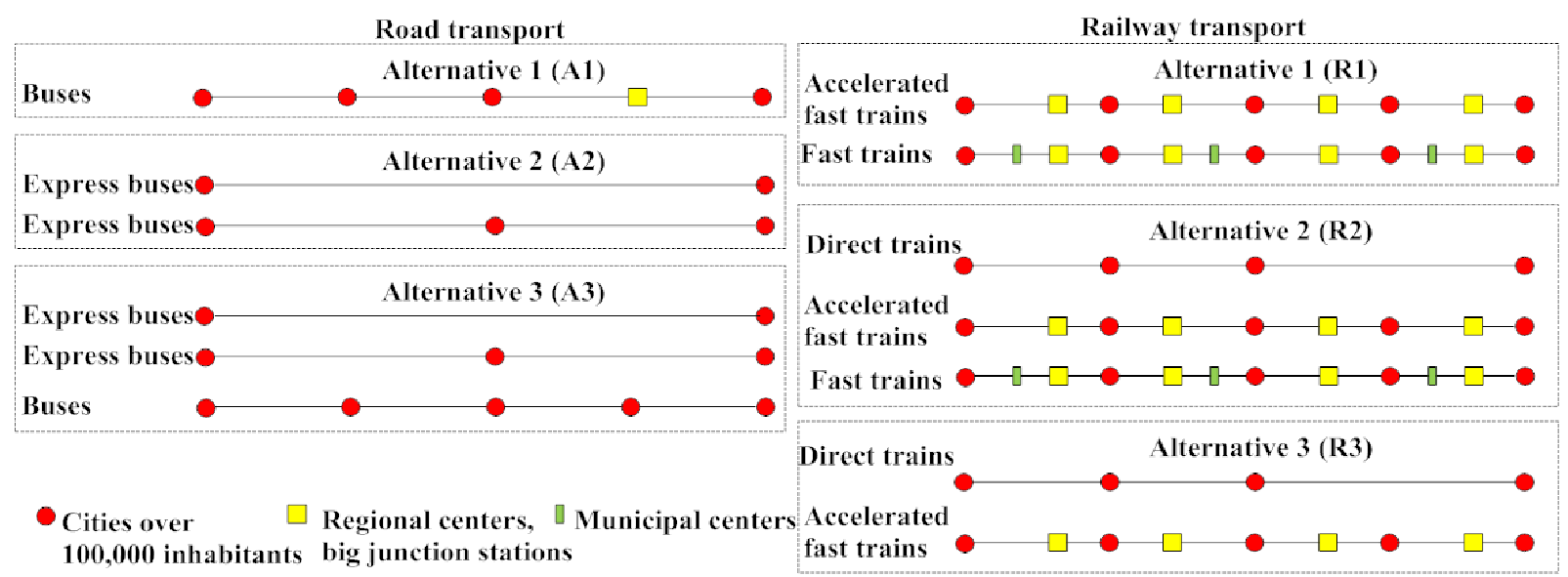

Fig. 1. Alternatives by road and railway transport

\section{Game theory model}

The game-theoretic formalization presented in the study allows modelling strategies and decision making for both railway and road operators in the case of different interests. Table 3 shows the payoff matrix, which presents the profit of the road operator. Each element of the matrix is determined by multiplication the number of passengers by the ticket price for road transport. It should take into account that there is difference between the ticket price for buses and express buses.

Table 3

Payoff matrix, EUR

\begin{tabular}{|c|c|c|c|c|}
\hline \multicolumn{3}{|c|}{$\begin{array}{c}\text { Player road } \\
\text { transport }\end{array}$} & \multicolumn{3}{|c|}{ Player railway transport - Strategies } \\
\cline { 2 - 4 } & $\mathrm{R} 1$ & $\mathrm{R} 2$ & $\mathrm{R} 3$ \\
\hline Strategies & Respective probabilities & $y_{1}$ & $y_{2}$ & $y_{3}$ \\
\hline A1 & $x_{1}$ & 6630 & 6120 & 6375 \\
\hline A2 & $x_{2}$ & 2754 & 2203 & 2478 \\
\hline A3 & $x_{3}$ & 9384 & 8323 & 8853 \\
\hline
\end{tabular}

The transformation for the player road transport according to Table 1 is as follows:

$$
\text { Minimize } \zeta=X_{1}+X_{2}+\ldots+X_{3} \text {, }
$$




$$
\mid \begin{gathered}
6630 X_{1}+2754 X_{2}+9384 X_{3} \leq 1 \\
6120 X_{1}+2203 X_{2}+2478 X_{3} \leq 1 \\
6275 X_{1}+2478 X_{2}+8853 X_{3} \leq 1 \\
X_{i} \geq 0, i=1,2, \ldots, m
\end{gathered}
$$

The transformation for the player railway transport according to Table 2 is as follows:

$$
\begin{gathered}
\text { Maximize } \omega=Y_{1}+Y_{2}+\ldots+Y_{n}, \\
\mid \begin{array}{c}
6630 Y_{1}+6120 Y_{2}+6375 Y_{3} \leq 1 \\
2754 Y_{1}+2203 Y_{2}+2478 Y_{3} \leq 1 \\
9384 Y_{1}+8323 Y_{2}+8853 Y_{3} \leq 1 \\
Y_{j} \geq 0, j=1,2, \ldots, n
\end{array}
\end{gathered}
$$

The mathematical models presented by formulas (20-23) are solved by the dual linear programming method. This means that the optimal solution of one problem (player road transport) automatically gives the optimal solution of the other (player railway transport). Table 4 shows the results for the respective probabilities for strategies. It can be seen that the optimal strategy for the road operator is A3, i.e. transportation by buses and express buses. The optimal strategy for railway transport is transportation by direct trains, accelerated fast trains and fast trains.

Table 4

Payoff matrix in EUR

\begin{tabular}{|c|c|c|c|}
\hline \multicolumn{2}{|c|}{$\begin{array}{c}\text { Player road } \\
\text { transport }\end{array}$} & \multicolumn{2}{c|}{$\begin{array}{c}\text { Player railway } \\
\text { transport }\end{array}$} \\
\hline Strategies & Respective probabilities & Strategies & Respective probabilities \\
\hline A1 & $x_{1}=0$ & $\mathrm{R} 1$ & $y_{1}=0$ \\
\hline $\mathrm{A} 2$ & $x_{2}=0$ & $\mathrm{R} 2$ & $y_{2}=1$ \\
\hline $\mathrm{A} 3$ & $x_{3}=1$ & $\mathrm{R} 3$ & $y_{3}=0$ \\
\hline \multicolumn{3}{|c|}{ The value of the game $\vartheta$ is 8323 EUR } \\
\hline
\end{tabular}

\section{Game model based on passengers}

In this case the payoff matrix is formed based on the number of passengers who were attracted by the road operator. In the game model these passengers are a loss for the railway operator. Table 5 presents the payoff matrix.

Table 5

Payoff matrix, passengers per day

\begin{tabular}{|c|c|c|c|c|}
\hline \multicolumn{2}{|c|}{$\begin{array}{c}\text { Player road } \\
\text { transport }\end{array}$} & \multicolumn{3}{c|}{ Player railway transport - Strategies } \\
\cline { 2 - 4 } & $\mathrm{R} 1$ & $\mathrm{R} 2$ & $\mathrm{R} 3$ \\
\hline Strategies & Respective probabilities & $y_{1}$ & $y_{2}$ & $y_{3}$ \\
\hline A1 & $x_{1}$ & 520 & 200 & 720 \\
\hline A2 & $x_{2}$ & 480 & 160 & 640 \\
\hline A3 & $x_{3}$ & 500 & 180 & 680 \\
\hline
\end{tabular}

The transformation for the player road transport according to Table 5 is as follows:

$$
\begin{gathered}
\text { Minimize } \zeta=X_{1}+X_{2}+X_{3}, \\
\mid \begin{array}{c}
520 X_{1}+200 X_{2}+720 X_{3} \leq 1 \\
480 X_{1}+160 X_{2}+640 X_{3} \leq 1 \\
500 X_{1}+180 X_{2}+680 X_{3} \leq 1 \\
X_{i} \geq 0, i=1,2, \ldots, m
\end{array}
\end{gathered}
$$


The transformation for the player railway transport according to Table 2 is as follows:

$$
\begin{gathered}
\text { Maximize } \omega=Y_{1}+Y_{2}+\ldots+Y_{n}, \\
\mid \begin{array}{c}
520 Y_{1}+480 Y_{2}+500 Y_{3} \leq 1 \\
200 Y_{1}+160 Y_{2}+180 Y_{3} \leq 1 \\
720 Y_{1}+640 Y_{2}+680 Y_{3} \leq 1 \\
Y_{j} \geq 0, j=1,2, \ldots, n
\end{array}
\end{gathered}
$$

Table 6 shows the results. It can be seen that the results are similar to the game model based on the profit of the road operator (Table 4). This shows the adequacy of both models.

Results for payoff matrix, passengers per day

\begin{tabular}{|c|c|c|c|}
\hline \multicolumn{2}{|c|}{$\begin{array}{c}\text { Player road } \\
\text { transport }\end{array}$} & \multicolumn{2}{c|}{$\begin{array}{c}\text { Player railway } \\
\text { transport }\end{array}$} \\
\hline Strategies & Respective probabilities & Strategies & Respective probabilities \\
\hline $\mathrm{A} 1$ & $x_{1}=0$ & $\mathrm{R} 1$ & $y_{1}=0$ \\
\hline $\mathrm{A} 2$ & $x_{2}=0$ & $\mathrm{R} 2$ & $y_{2}=1$ \\
\hline $\mathrm{A} 3$ & $x_{3}=1$ & $\mathrm{R} 3$ & $y_{3}=0$ \\
\hline \multicolumn{2}{|c|}{ The value of the game $\vartheta$ is 640 passengers } \\
\hline
\end{tabular}

The both game models can be drawn up based on the losses for the railway operator. The results will be the same according to the dual linear programming method.

\section{Verification of the results}

The verification of the results has been conducted by the Hurwitz's criterion. The road operator (strategies A1, A2, A3) aims to choose the strategy with the highest profit. Table 7 presents the values of the Hurwitz's criterion according to formula (19). The first part of the table presents the decision matrix for the road operator given in Table 3. The railway operator (strategies R1, R2, R3) aims to choose the strategy with the lowest cost.

Table 8 shows the values of the Hurwitz's criterion according to formula (18). The interests of both operators are opposite. The decision matrix for the railway operator is formed as a transposed matrix of that of the road operator, since the profit of the road operator is a loss for the railway operator.

Figure 2 and Figure 3 show the results for the Hurwitz's criterion at different values of the coefficient $\alpha$. It can be seen that the optimal strategy for the road operator is A3 (transportation by buses and express buses), since it has the maximum value for the Hurwitz's criterion for all values of the coefficient $\alpha$. The optimal strategy for the railway operator is strategy R2 (transportation by direct trains, accelerated fast trains and fast trains), since it has the minimum value for the Hurwitz's criterion for all values of the coefficient $\alpha$.

It can be concluded that the results of the application of the Hurwitz's criterion give the same results as those obtained from the game theory.

Hurwitz's criterion for player A

\begin{tabular}{|c|c|c|c|c|c|c|c|c|c|c|c|c|c|c|}
\hline \multicolumn{4}{|c|}{ Decision matrix } & \multicolumn{11}{|c|}{ Hurwitz's criterion } \\
\hline \multirow{2}{*}{ Road } & \multicolumn{3}{|c|}{ Rail } & \multicolumn{11}{|c|}{ Coefficient $\alpha$} \\
\hline & R1 & $\mathrm{R} 2$ & R3 & 0 & 0.1 & 0.2 & 0.3 & 0.4 & 0.5 & 0.6 & 0.7 & 0.8 & 0.9 & 1 \\
\hline A1 & 6630 & 6120 & 6375 & 6120 & 6171 & 6222 & 6273 & 6324 & 6375 & 6426 & 6477 & 6528 & 6579 & 6630 \\
\hline A2 & 2754 & 2203 & 2478 & 2203 & 2258 & 2313 & 2368 & 2423 & 2479 & 2534 & 2589 & 2644 & 2699 & 2754 \\
\hline A3 & 9384 & 8323 & 8853 & 8323 & 8429 & 8535 & 8641 & 8747 & 8854 & 8960 & 9066 & 9172 & 9278 & 9384 \\
\hline
\end{tabular}

Table 7 
Hurwitz's criterion for player B

\begin{tabular}{|c|c|c|c|c|c|c|c|c|c|c|c|c|c|c|}
\hline \multicolumn{1}{|c|}{ Decision matrix } & \multicolumn{10}{c|}{ Hurwitz's criterion } \\
\hline \multirow{2}{*}{ Rail } & \multicolumn{3}{|c|}{ Road } & \multicolumn{10}{c|}{ Coefficient $\alpha$} \\
\cline { 2 - 17 } & A1 & A2 & A3 & 0 & 0.1 & 0.2 & 0.3 & 0.4 & 0.5 & 0.6 & 0.7 & 0.8 & 0.9 & 1 \\
\hline R1 & 6630 & 2754 & 9384 & 2754 & 3417 & 4080 & 4743 & 5406 & 6069 & 6732 & 7395 & 8058 & 8721 & 9384 \\
\hline R2 & 6120 & 2203 & 8323 & 2203 & 2815 & 3427 & 4039 & 4651 & 5263 & 5875 & 6487 & 7099 & 7711 & 8323 \\
\hline R3 & 6375 & 2478 & 8853 & 2478 & 3115 & 3753 & 4390 & 5028 & 5665 & 6303 & 6940 & 7578 & 8215 & 8853 \\
\hline
\end{tabular}

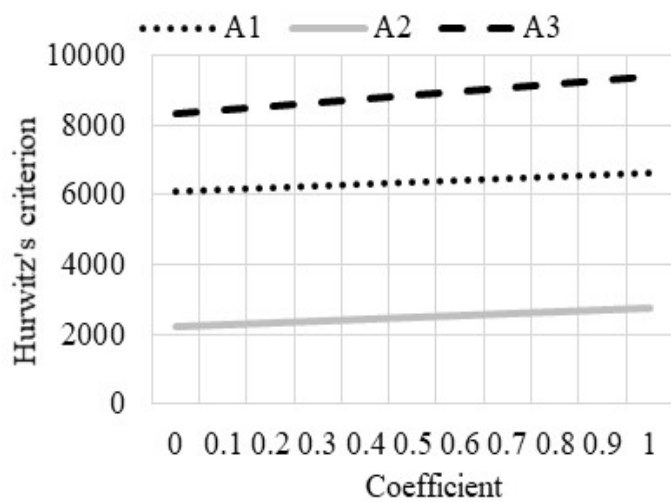

Fig. 2. Ranking the alternatives for player $\mathbf{A}$

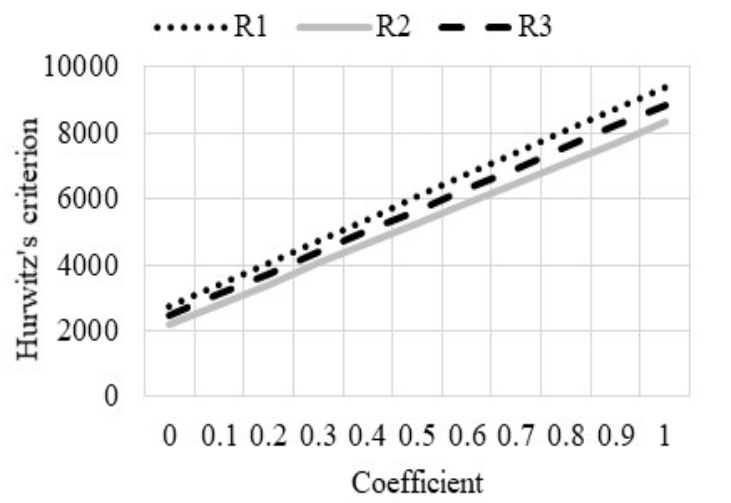

Fig. 3. Ranking the alternatives for player $B$

\section{Conclusions}

In this research an approach has been developed based on the game theory for planning passenger rail and road transport on parallel routes. Two game models have been developed, one based on the profit of the road operator and the other based on the number of passengers. The strategies for both operators have been studied. The both models have similar results. The results propose the strategies for transport planning for both operators. It was found that for the road transport the optimal strategy is carriage by buses and express buses. For the railway transport the optimal transport plan includes carriage by fast trains, accelerated fast trains and direct trains.

The results of the game theory have been verified by the Hurwitz's criterion. It was found that the results of both approaches are similar. The verification of the results shows the applicability of the developed methodology.

The game model based on passengers could be used for initial research; the model based on the profit could serve to study variability of optimal strategies, depending on the bus operator profit (ticket price).

The game-theoretic formalization presented in the study allows modelling strategies and decision making for both railway and road operators in the case of different interests. The proposed methodology can be used in planning passenger rail and road transport on parallel routes and also making decisions in transport management.

\section{Acknowledgements}

This research has been supported by the contract No.KП-06-H27/12 of 11.12.2018 "Modelling and elaboration of complex system for selection of transport technology in transport network" funded by the National Science Fund of the Ministry of Education and Science of Bulgaria.

\section{References}

[1] Yang L., Y. Shi, S., Hao, L. Wu. Route Choice Model Based on Game Theory for Commuters. Promet - Traffic\&Transportation, Vol. 28, 2016, No. 3, pp. 195-203. 
[2] Hernández R., Cárdenas C., Muñoz D. Game theory applied to transportation systems in Smart Cities: Analysis of evolutionary stable strategies in a generic carpooling system, International Journal on Interactive Design and Manufacturing, 12, 2018, pp. 179-185.

[3] Koryagin M. E. Game theory approach to optimizing of public transport traffic under conditions of travel mode choice by passengers. Transport Problems, 9, 2014, pp. 117-124.

[4] Koryagin M.E. Urban Planning: A Game Theory Application for the Travel Demand Management. PeriodicaPolytechnica Transportation Engineering, 46(4), 2018, pp. 171-178.

[5] Xiaowei HU, Wang J, and Guanglin SUN. A Game theory approach for the operators' behavior analysis in the urban passenger transportation market.Advanced Engineering Forum Online, Vol. 5, 2012, pp. 38-43.

[6] Hollander Y, Prashker J N. The applicability of non-cooperative game theory in transport analysis. Transportation, 33:481, 2006,496.

[7] Roumboutsos A.,KaprosS., A game theory approach to urban public transport integration policyTransport Policy 15, 2008, pp. 209-215.

[8] Śkrinjar J, Abramović B, Brnjac N. The use of game theory in urban transport planning,Tehničkivjesnik 22, 6, 2015, pp. 1617-1621.

[9] Wu C., Pei Y., Gao J. Evolution Game Model of Travel Mode Choice in Metropolitan., Discrete Dynamics in Nature and Society, Vol. 2015, Article ID 638972, 11 p.

[10]Lian-Ju Sun, Zi-You Gao. An equilibrium model for urban transit assignment based on game theory. European Journal of Operational Research, 181, 2007, pp. 305-314.

[11]Raturi V., Srinivasan K., Narulkar G., Chandrashekharaiah A., Gupta A. Analyzing inter-modal competition between high speed rail and conventional transport systems: A game theoretic approach. Procedia - Social and Behavioral Sciences, 104, 2013, pp. 904-913.

[12] Michael G.H. Bell. A game theory approach to measuring the performance reliability of transport networks.Transportation Research Part B, 34, 2000, pp. 533-545.

[13] Chiung-Wen Hsu, Yusin Lee, Chun-Hsiung Liao. Competition between high-speed and conventional rail systems: A game theoretical approach. Expert Systems with Applications 37. 2010, pp. 3162-3170.

[14] Taha H. Operations Research: An Introduction, 2011, 9th Edition Prentice Hall. 